\title{
BMJ Open Evidence for validity of a national physician and patient-reported, cross-sectional survey in China and UK: the Disease Specific Programme
}

\author{
S M Babineaux, ${ }^{1}$ B Curtis, ${ }^{1}$ T Holbrook, ${ }^{2}$ G Milligan, ${ }^{2} \mathrm{~J} \mathrm{Piercy}^{2}$
}

To cite: Babineaux SM, Curtis B, Holbrook T, et al. Evidence for validity of a national physician and patient-reported, cross-sectional survey in China and UK: the Disease Specific Programme. BMJ Open 2016;6:e010352. doi:10.1136/bmjopen-2015010352

- Prepublication history and additional material is available. To view please visit the journal (http://dx.doi.org/ 10.1136/bmjopen-2015010352).

Received 18 November 2015 Revised 12 May 2016 Accepted 20 July 2016

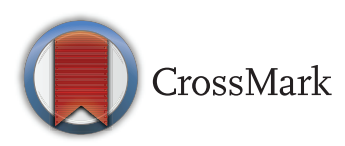

${ }^{1}$ Eli Lilly \& Co, Indianapolis, Indiana, USA

${ }^{2}$ Adelphi Real World,

Macclesfield, UK

Correspondence to

Dr J Piercy;

james.piercy@adelphigroup. com

\section{ABSTRACT}

Objective: Diabetes represents a significant challenge for Chinese healthcare providers. Healthcare decisionmaking is generally based on many data sources, including randomised controlled and real-world studies; however, good-quality data from Chinese diabetes patients are scarce. We performed an initial validation to assess the representativeness of one source of real-world data-the Diabetes Adelphi Disease Specific Programme (DSP) in China.

Setting: China, UK.

Participants: The Chinese DSP included 2060 patients with previously diagnosed type 2 diabetes mellitus (T2DM) sampled by 200 physicians. The reference Chinese population comprised 238639 patients with previously diagnosed T2DM. The UK DSP contained 1481 patients with T2DM sampled by 125 physicians; the reference UK population comprised 289 patients with diabetes.

Primary and secondary outcomes: The primary outcome was comparison of unweighted China DSP and reference data for sex, body mass index (BMI), blood pressure (BP), patients achieving glycosylated haemoglobin $\left(\mathrm{HbA}_{1 \mathrm{c}}\right)<7 \%$, total cholesterol, coronary heart disease and dyslipidaemia. The secondary outcome was comparison of weighted UK DSP and reference data for $\mathrm{BMI}, \mathrm{BP}$, mean $\mathrm{HbA}_{1 \mathrm{c}}$, total cholesterol, smoking and insulin status.

Results: Comparison of unweighted China DSP and reference data revealed statistical equivalence for $\mathrm{BMI}$, systolic $\mathrm{BP}$, proportion of patients achieving $\mathrm{HbA}_{1 \mathrm{C}}$ $<7 \%$, total cholesterol, coronary heart disease and dyslipidaemia. Sex, age, diabetes duration, diastolic BP and mean $\mathrm{HbA}_{1 \mathrm{c}}$ level were not equivalent, although differences were generally small. Weighting of data did not substantially affect the results. A similar pattern was observed for UK data.

Conclusions: This study provides evidence that the methodology used for the China and UK parts of the Diabetes DSP produces representative samples that are comparable with other independent sources of patient treatment outcomes data, which may ultimately inform public health decision-making. Although this method could be used in other countries, the current validation applies to UK and China. Further research is required for other countries.

\section{Strengths and limitations of this study}

- The Adelphi Real World Disease Specific Programme (DSP) is a valuable source of information on patients with type 2 diabetes in China, a region where reliable and up-to-date information is lacking.

- This analysis has demonstrated, by comparison with a large, reference population-based, crosssectional survey, that the DSP population is representative of patients with type 2 diabetes in China.

- The representativeness of the DSP population was further supported by comparison of the UK Diabetes DSP with diabetes data gathered in the Health Survey for England.

- Limitations of the study include the selection of patients included in the DSP samples, which depends on the physician's diagnostic skills, and the potential for over-representation of patients with more severe disease than the general population.

- Patient-level data were available for the DSP and Health Survey for England populations but not the Chinese reference population, for which only aggregate data were available; as a result, possible design bias could not be addressed in the Chinese reference population.

\section{INTRODUCTION}

The International Diabetes Federation estimated the national prevalence of diabetes to be $9.32 \%$ for China in $2014,{ }^{1}$ a significant increase from the $<1 \%$ prevalence reported in $1980 .^{2}$ This translated into an estimated 96 million individuals with diabetes and 1.2 million diabetes-related deaths in $2014 .{ }^{1}$ This represents a significant challenge; public health planners making formulary and reimbursement decisions must decide how to meet changing priorities by efficiently allocating funding and ensuring appropriate access to medicines. To date, treatment guidelines have largely been based on 
evidence from non-Asian populations, although an increasing number of randomised clinical trials are now in progress in China. ${ }^{3}$

Currently, decisions regarding the availability and reimbursement of medicines are made at the government, regional, local and hospital level in China. Decision-makers have differing evidentiary requirements and varied data are required to support the value of specific interventions. Data need to be current, treatmentspecific, valid at a patient level, relevant and obtained from a representative sample. Outcomes data are also required, including safety and drug surveillance information, and efficacy, cost and resource-use data. Such data may be scarce and not readily available.

Although considered the gold standard for questions relating to efficacy and safety, data from randomised controlled studies are often unrepresentative of the population in which the intervention will be used because of strict inclusion criteria. Renal and cardiovascular complications may lead to the exclusion of many patients with diabetes from randomised clinical studies. ${ }^{4}$ For example, only half of Finnish patients with diabetes beginning treatment with statins for diabetic dyslipidaemia would have qualified for inclusion in the Heart Protection Study and the Collaborative Atorvastatin Diabetes Study. ${ }^{5}$ Epidemiological or 'real-world' studies provide information on larger, more representative populations but are generally not accessible to patientlevel interrogation.

One source of real-world data for Chinese patients with diabetes is the Adelphi Real World Disease Specific Programme (DSP) for diabetes. DSPs, which are cross-sectional surveys generating data from real-world clinical practice, collect current patient demographic data and treatment practices, in addition to resource-use and quality-of-life data, in specific therapy areas and meet the majority of the criteria described above. ${ }^{6}$ DSPs have been conducted for a variety of therapy areas, including diabetes, in countries with varying healthcare systems and following societal changes, such as seen in China.

One important consideration of DSP validation is determining the representativeness of the data compared with wider populations. With that aim, we compared DSP diabetes data for China against a reference Chinese data source. To support this analysis, we performed a similar validation of the DSP in a developed western market with a contrasting socioeconomic and healthcare system to China, to demonstrate the adaptability of the data collection methodology. We selected the UK for this confirmatory validation because the availability of a reliable reference data source made it possible to assess the representativeness of DSP diabetes data for the UK compared with the wider UK population. We hypothesised that, viewed together, these analyses would provide evidence for the representativeness of the DSP as a source of real-world evidence for patients with diabetes in China.
METHODS

\section{Disease Specific Programmes}

DSPs are large, multinational surveys of clinical practice that describe current disease management, diseaseburden impact and associated treatment effects (patientreported, clinical and physician-reported). The survey method is designed to adapt to any country, culture or disease area, with rapid implementation facilitating collection of up-to-date data. DSPs collect qualitative and quantitative data from four key sources of information: physician interviews, physician workload questionnaires, patient record forms completed by the doctor and questionnaires completed by the same patients. Physicians are selected for participation based on their eligibility to participate in the DSP in terms of specialty, location (hospital or office), whether they are personally responsible for treatment decisions and how many patients they see in a typical week. Candidate physicians who meet these criteria are invited to participate in the DSP; those who agree to participate are reimbursed for their time according to national reimbursement rates in their country. Patients are recruited only once and have no further follow-up as each DSP is a point-in-time survey. DSPs are repeated every 1 or 2 years, depending on the disease area, introduction of new treatments and how often guidelines are updated. The stages of DSP development are summarised in online supplementary figure S1; full details of DSP methodology have been published previously. ${ }^{6}$

The Diabetes DSPs selected for this analysis were conducted in China in 2012 and in the UK in 2013; these versions were chosen to match the time of data collection for the reference data sources. Geographically representative primary care physicians and specialists (hospital physicians only in China) were asked to sample the next 10 patients presenting with type 2 diabetes mellitus (T2DM), aged $>18$ years and currently taking antidiabetic drugs. Additional criteria that applied specifically to the present analysis, over and above those used to recruit patients for the DSP and in order to match the criteria of the comparator study, were that patients were not to be presenting for the first time with T2DM and insulin monotherapy was not allowed. Physicians completed a patient record form for these patients and gave them a patient self-completion form. Physicians recorded information on patient demographics, clinical characteristics (including glycaemic control and hypoglycaemic events in the Diabetes DSP), medications administered and resource use. The questions used in this analysis in China and the UK are shown in online supplementary appendices 1 and 2, respectively. Although not used in this analysis, the voluntary patient self-completion forms collected information about how diabetes affected the patient's everyday life, together with their opinions and understanding of their medications and glycaemic control. Patients could complete any or none of the questions and were instructed to complete the form without help from their healthcare practitioner. 
The DSP is research involving survey procedures and as such does not require ethics committee approval. Patients provide informed consent for use of their anonymised and aggregated data for research and publication in scientific journals. This is achieved by means of a check box on the front page of the patient-completed survey. Data are collected in such a way that patients and physicians cannot be identified directly, with all data being aggregated and de-identified before receipt by Adelphi Real World. DSPs are performed in accordance with the European Pharmaceutical Marketing Research Association (EphMRA) guidelines ${ }^{7}$ and the US Health Insurance Portability and Accountability Act $1996 .{ }^{8}$

\section{Comparator data}

The Chinese reference data set was based on a multicentre, cross-sectional survey of outpatients with T2DM in 606 hospitals across China between April and June 2011. ${ }^{9}$ The first seven patients entering the facilities and meeting the following criteria were included: diagnosed with T2DM; one or more previous outpatient medical record pertaining to diabetes; aged >18 years; and treated with oral antidiabetic agents, either alone, with insulin or with glucagon-like peptide-1 agonists.

The UK Diabetes DSP data were compared against the Health Survey for England (HSFE) 2011 (10 617 patients). ${ }^{10}$ This is a cross-sectional, country-wide health survey that monitors health trends across the general population, estimating proportions of people with specified health conditions and the prevalence of risk factors and combinations of risk factors associated with these conditions. The 2011 HSFE had a special focus on cardiovascular disease, hypertension and diabetes, and included diabetes-related variables, for example, age at diagnosis and insulin treatment, in addition to standard demographic data and information on other disease areas. The HSFE included patients taking antidiabetic medication and those managing their condition with insulin alone or with diet and exercise alone; we applied exclusion criteria to identify those with T2DM who were treated with an antidiabetic medication.

To ensure comparability of the UK DSP and HSFE populations, patient characteristics were matched as closely as possible. This required exclusion of patients aged $\leq 18$ years; diagnosed before age 35 years and treated with insulin (a proxy for type 1 diabetes in the absence of an explicit indicator in this database); and those not receiving endocrine drug treatment (a broad term used in the HSFE coding system ${ }^{11}$ and used in the current analysis to exclude patients managing their condition with diet and exercise). Pregnant women were also excluded.

\section{Statistical analysis}

Clinical and demographic characteristics common to the China DSP and Chinese reference population and the UK DSP and the HSFE data were compared to assess the validity of the UK and China DSPs. In order to compare the DSP and reference populations, weighting was needed to correct for imbalances between the groups. In the DSPs, patients consulting more frequently have a greater chance of selection and for a given frequency of visiting, a patient's chance of being sampled is a function of the total number of patients managed by the doctor, that is, the more patients a doctor manages, the less likely it is that any individual will be sampled. Inverse probability weighting was used to account for this, incorporating the frequency of visits made by the patient in the last 12 months (adjusted to 12 months if the patient had been managed for $<12$ months) and the total number of patients managed by each doctor (in conjunction with the number of patients sampled by each doctor). A random sample of patients with T2DM would include very few patients diagnosed on the same day as the study sampling, whereas the DSP population contained a relatively high number of patients attending for initial diagnosis because patients were sampled on days when they consulted their doctor. To better approximate a random sample, as well as matching the inclusion criteria for the comparator study in China, patients diagnosed on the day of the sample were excluded from the weighted analysis. The HSFE 2011 used a clustered stratified multistage sampling design. Similar to the weighting applied to the DSP, weights were applied to the HSFE data, according to guidance issued by the HSFE, to account for selection and nonresponse bias. ${ }^{12}$ Missing data were assumed to be missing at random and were not imputed.

Standard tests, such as the t-test and $\chi^{2}$ test, assume a null hypothesis that the two comparator groups are the same. Only if the $\mathrm{p}$ value is $<0.05$ can that hypothesis be rejected and a significant difference be claimed. A $\mathrm{p}$ value $\geq 0.05$ does not allow the claim that there is no difference. Therefore, standard tests were not appropriate in this analysis, where the aim was to show no difference and tests for 'equivalence' were required. Variables common to each pair of data sets were compared using two one-sided tests aimed at testing for equivalence. ${ }^{13}$ Two means are considered equivalent if they occur within a predefined 'distance' or tolerance of each other. A sensible tolerance is the minimum important difference (MID). If the MID is unknown, assuming an MID of $25-50 \%$ of the overall SD is considered reasonable. ${ }^{14}$ In the present analysis, an MID of $25 \%$ was assumed for all variables. For proportions of patients (eg, proportion with hypertension), an MID of $25 \%$ of that proportion was used.

\section{RESULTS}

\section{China}

The Chinese DSP included 2060 patients with T2DM sampled by 200 physicians. A total of 398 patients were receiving insulin only and were excluded in line with the reference population; the Chinese unweighted DSP 
population therefore included 1662 patients with T2DM. Table 1 shows clinical and demographic variables collected in both surveys. Patients' mean body mass index (BMI) was on the upper limit of normal at $24.3 \mathrm{~kg} / \mathrm{m}^{2}$. Mean diastolic blood pressure (DBP) and systolic blood pressure (SBP) were high $(83.2$ and $132.7 \mathrm{~mm} \mathrm{Hg}$, respectively), mean glycosylated haemoglobin $\left(\mathrm{HbA}_{1 \mathrm{c}}\right)$ level was high $(7.4 \%)$ and the proportion of patients with $\mathrm{HbA}_{1 \mathrm{c}}<7 \%$ was low (33\%). Weighting of data to account for DSP design bias led to the exclusion of 79 patients but did not substantially change any disease characteristics other than the proportion of patients with comorbidities.

The reference Chinese population comprised 238639 patients with T2DM. Patients had a mean age of 58.7 years, mean BMI of $24.4 \mathrm{~kg} / \mathrm{m}^{2}$ and mean diabetes duration of 5.6 years (table 1). Mean DBP and SBP were high (81.0 and $131.9 \mathrm{~mm} \mathrm{Hg}$, respectively); mean total cholesterol level was normal at $183 \mathrm{mg} / \mathrm{dL}$, although mean $\mathrm{HbA}_{1 \mathrm{c}}$ level was high $(7.9 \%)$ and the proportion of patients with $\mathrm{HbA}_{1 \mathrm{c}}<7 \%$ was low $(32 \%)$.

Comparison of the unweighted China DSP and Chinese reference populations revealed statistical equivalence for BMI, SBP and the comorbidities coronary heart disease and dyslipidaemia (table 1). Variables for which there was not enough evidence for equivalence were sex, age, duration of diabetes, $\mathrm{DBP}$ and $\mathrm{HbA}_{1 \mathrm{c}}$ level, although the proportion of patients achieving an $\mathrm{HbA}_{1 \mathrm{c}}$ level $<7 \%$ was equivalent in both populations. Weighting of the DSP data did not substantially affect these differences.

\section{United Kingdom}

The UK DSP contained 1481 patients with T2DM sampled by 125 physicians; 1213 patients were eligible for inclusion in the analysis of unweighted data. In total, 268 patients were not eligible because they had type 1 diabetes $(n=244)$, were diagnosed before the age of 35 years and treated with insulin only $(n=19)$ or were pregnant $(n=5)$. Weighting resulted in exclusion of another 41 patients who were diagnosed with diabetes on the day of the survey; the weighted analysis population thus comprised 1172 patients.

Overall, 8610 adults and 2007 children were interviewed for the 2011 HSFE; 2206 were excluded as they were under the age of 18 years, 7878 did not have a diagnosis of diabetes and 244 were not receiving an endocrine agent and were also excluded. The UK reference population therefore comprised 289 patients. Variables collected in both surveys are given in table 2 . Total cholesterol levels were normal in both groups, as was DBP. SBP and BMI were high in both groups.

Comparison of the unweighted UK DSP and HSFE populations revealed statistical equivalence in sex, age at diagnosis, BMI and total cholesterol level (table 2). Some exceptions, where equivalence could not be demonstrated, were observed. DSP patients were younger, had a shorter time since diagnosis, lower
$\mathrm{HbA}_{1 \mathrm{c}}$ level and higher SBP and DBP than HSFE patients. Weighting of the UK DSP population did not substantially affect these differences, with the exception of total cholesterol level, which became statistically equivalent after weighting.

\section{DISCUSSION}

Public health planners in China face a diabetes epidemic and must make treatment recommendations complicated by a paucity of good-quality data obtained in relevant populations in a timely manner. While collection and reporting of data is improving, data are scarce that satisfy all the criteria required to meet the needs of Chinese decision-makers. DSPs offer one solution to this issue, being sufficiently up to date, collected rapidly and frequently and containing information on a breadth of clinical, demographic and outcome variables that can inform public health decision-making when used with other supporting data.

The current analysis was undertaken to demonstrate the representativeness of DSP data compared with the Chinese T2DM population. Comparison of the China DSP and reference Chinese populations identified equivalence in many variables common to both studies. Some areas of non-equivalence were observed: time since diagnosis of diabetes was longer in patients in the reference population and DBP values were non-equivalent, although the difference was small $(<2 \mathrm{~mm} \mathrm{Hg})$ and its clinical relevance questionable. Mean $\mathrm{HbA}_{1 c}$ level was non-equivalent, although the proportion of patients achieving $\mathrm{HbA}_{1 \mathrm{c}}<7 \%$ was equivalent. This suggests that the reference data set may have contained more patients with high values, which would have a greater impact on mean $\mathrm{HbA}_{1 \mathrm{c}}$ than on the proportion with $\mathrm{HbA}_{1 \mathrm{c}}<7 \%$. The between-group difference is within the bounds of natural variation, as reflected by the SDs and the mean baseline $\mathrm{HbA}_{1 \mathrm{c}}$ levels of $7.0-8.3 \%$ reported in other observational and phase IV studies or surveys. ${ }^{15-17}$

The comparison of UK DSP and HSFE diabetes data, which was performed to substantiate the findings of the Chinese comparison, also provided evidence for the representativeness of the DSP, with equivalence in many of variables. Although equivalence was not demonstrated for some, including patient age and time since diagnosis, this may reflect different characteristics of presenting patients in the DSP versus the randomly selected HSFE group. $\mathrm{HbA}_{1 \mathrm{c}}$ and SBP were non-equivalent, although between-group differences were small $(<0.2 \%$ and $2.5 \mathrm{~mm} \mathrm{Hg}$, respectively), within the bounds of natural variation as reflected by the SDs and of questionable clinical relevance. $^{18} 19$

In line with other observational and real-world studies, several limitations of the data sources should be considered. The primary limitations of the DSP relate to selection and diagnosis of patients. Physicians were asked to include the next 10 presenting patients with T2DM to reduce selection bias. The integrity of this process 
Table 1 Comparison of the Chinese Diabetes DSP and Chinese reference populations

\begin{tabular}{|c|c|c|c|c|c|c|c|}
\hline \multirow[b]{2}{*}{ Variable } & \multirow{2}{*}{$\begin{array}{l}\text { Chinese reference } \\
\text { population } \\
(n=238639)\end{array}$} & \multicolumn{2}{|c|}{ DSP unweighted } & \multirow[b]{2}{*}{ p Value* } & \multicolumn{2}{|l|}{$\begin{array}{l}\text { DSP } \\
\text { weighted } \dagger\end{array}$} & \multirow[b]{2}{*}{ p Value ${ }^{\star}$} \\
\hline & & $\mathrm{n}=1662$ & Missing & & $n=1583$ & Missing & \\
\hline Male, \% & 52.2 & 47.7 & 0 & 1.0000 & 46.7 & 0 & 1.000 \\
\hline Mean age, years (SD) & $58.7(11.7)$ & $56.1(11.3)$ & 0 & 0.1423 & $56.4(11.1)$ & 0 & 0.0963 \\
\hline Mean time since diagnosis, years (SD) & $5.6(5.3)$ & $3.3(3.6)$ & 34 & 1.0000 & $3.5(3.5)$ & 34 & 1.000 \\
\hline Mean BMI, $\mathrm{kg} / \mathrm{m}^{2}$ (SD) & $24.4(3.2)$ & $24.3(3.1)$ & 6 & 0.0000 & $24.5(3.2)$ & 6 & 0.0000 \\
\hline Mean total cholesterol level, mg/dL (SD) & $182.9(57.2)$ & $185.4(39.6)$ & 399 & 0.0000 & $186.9(41.5)$ & 379 & 0.0000 \\
\hline Mean $\mathrm{HbA}_{1 \mathrm{c}}$ level, \% (SD) & $7.9(1.7)$ & $7.4(1.0)$ & 73 & 1.0000 & $7.3(1.0)$ & 71 & 0.9990 \\
\hline $\mathrm{HbA}_{1 \mathrm{c}}<7 \%, \%$ & 31.8 & 32.8 & 73 & 0.0000 & 33.3 & 71 & 0.0000 \\
\hline Mean diastolic BP, mm Hg (SD) & $81.0(11.1)$ & $83.2(8.6)$ & 54 & 0.0962 & $83.1(8.1)$ & 51 & 0.1439 \\
\hline Mean systolic BP, mm Hg (SD) & $131.9(15.1)$ & $132.7(12.3)$ & 54 & 0.0000 & $133.4(11.8)$ & 51 & 0.0000 \\
\hline \multicolumn{8}{|l|}{ Comorbidities, \% } \\
\hline Coronary heart disease & 10.9 & 9.2 & 0 & 0.0000 & 8.8 & 0 & 0.0000 \\
\hline Dyslipidaemia & 19.7 & 22.8 & 0 & 0.0000 & 25.6 & 0 & 0.0193 \\
\hline
\end{tabular}

Values in bold are $p>0.05$, that is, evidence is not strong enough to show equivalence.

* $p$ Value for comparison with Chinese reference population.

†Weighted to account for design bias in the DSP.

$\mathrm{BMI}$, body mass index; BP, blood pressure; DSP, Disease Specific Programme; $\mathrm{HbA}_{1 \mathrm{c}}$, glycosylated haemoglobin.

depends on the physician's diagnostic skills as no formal entry criteria were specified. In addition, this process favours patients presenting more frequently, as more frequent consultation increases the likelihood of selection. Although we corrected for this using a weighting process, the possibility cannot be excluded that patients with more severe disease or complications might be overrepresented in the DSP population. No patient-level data were available for the Chinese reference source and aggregated evidence was used instead. As a result, design bias could not be corrected through weighting. This means that even if the DSP data were perfectly corrected to be representative of the Chinese diabetes population, some differences would still be observed. We noted differences between the DSP and reference populations that were not corrected either by looking only at the prevalent population or by weighting; further research is required to identify potential reasons for such differences. The HSFE also has limitations, including oversampling in underpopulated areas, lack of response and differences in how study visits and procedures were performed. These limitations were addressed using a complex weighting strategy. Potential bias relating to non-response as a result of ill health was not, however, accounted for in the HSFE. These limitations must be taken into consideration before generalising the findings to other populations.

The strengths of the DSP approach should be considered. Although the DSPs are exploratory studies that complement rather than replace larger studies, advantages include the ability to rapidly perform studies in relatively small populations that nonetheless provide insights into diseases, attitudes and outcomes that might otherwise be difficult to obtain in such a timely manner

Table 2 Comparison of UK Diabetes DSP and UK reference HSFE populations

\begin{tabular}{|c|c|c|c|c|c|c|c|}
\hline \multirow[b]{2}{*}{ Variable } & \multirow[b]{2}{*}{ HSFE $(n=289)$} & \multicolumn{2}{|c|}{ DSP unweighted } & \multirow[b]{2}{*}{ p Value } & \multicolumn{2}{|c|}{ DSP weighted* } & \multirow[b]{2}{*}{ p Value } \\
\hline & & $n=1213$ & Missing & & $\mathrm{n}=1172$ & Missing & \\
\hline Male, \% & 58.2 & 58.4 & 0 & 0.0001 & 57.7 & 0 & 0.0003 \\
\hline Mean age at diagnosis, years (SD) & $54.1(14.1)$ & $54.6(11.5)$ & 98 & 0.001 & $54.7(11.5)$ & 98 & 0.0027 \\
\hline Mean time since diagnosis, years (SD) & $9.7(9.1)$ & $6.9(6.2)$ & 87 & 0.989 & $7.3(6.1)$ & 87 & 0.925 \\
\hline Current smoker, \% & 14.3 & 16.1 & 32 & 0.002 & 15.7 & 32 & 0.0018 \\
\hline Mean total cholesterol level, mg/dL (SD) & $172.1(51.1)$ & $178.3(55.9)$ & 121 & 0.0619 & $176.7(54.7)$ & 101 & 0.0349 \\
\hline Mean $\mathrm{HbA}_{1 \mathrm{c}}$ level, \% (SD) & $8.0(1.6)$ & $7.8(1.7)$ & 17 & 0.0754 & $7.8(1.7)$ & 13 & 0.063 \\
\hline Mean diastolic BP, mm Hg (SD) & $71.1(11.3)$ & $76.8(9.6)$ & 216 & 1.000 & $76.7(9.5)$ & 206 & 1.000 \\
\hline Mean systolic BP, mm Hg (SD) & $132.6(16.5)$ & $134.9(15.4)$ & 216 & 0.070 & $135.1(15.6)$ & 206 & 0.109 \\
\hline
\end{tabular}


and at in-depth patient level. A consistent methodology is used for DSPs across countries and economic environments, enabling cross-country comparisons. This may not be possible using registries or databases designed to be specific for a particular country or region. DSPs can also include elements related to patient-reported outcomes and impact on usual activities, providing insights into aspects not routinely assessed in randomised clinical trials.

Observational studies, such as performed by Ji et $a l^{9}{ }^{9}$ provide important epidemiological information in rapidly changing healthcare environments. This reference population comprised patients from 606 hospitals representing every region of mainland China other than Tibet and was designed to represent all regions of the Chinese mainland. Other strengths include the population size and census-style data. All patients were consulting a physician, similar to the DSP, and physicians sampled consecutive presenting patients in both studies. The strength of the HSFE lies in its epidemiological robustness in terms of general population coverage and collection of comprehensive clinical data and patientreported symptom and burden outcomes from a representative cross section of England, repeated annually with a consistent methodology. ${ }^{10}$ Both sources complement the DSPs as a result of consistency of overlapping variables; the depth of information provided by the DSPs on a smaller number of patients also complements the larger but less detailed data presented by Ji $e t a t^{p}$ and the HSFE. ${ }^{10}$

Ultimately, no single source provides all the data needed by every stakeholder. The China DSP and reference study are complementary and valid as variables common to both studies are consistent. Importantly, the independence of each study allows validation of the other. The HSFE and UK DSP have also been shown to be complementary, each validating the other. The DSPs can therefore be used to complement data from clinical trials performed in well-defined but potentially unrepresentative populations to provide an update on data otherwise obtained from large-scale but costly and timeconsuming epidemiological studies. Based on the results of the present analysis and considering the limitations discussed above, the Chinese and UK DSP data could be considered appropriate for inclusion in submissions for health technology assessments. While the DSPs are a useful additional tool for modelling and health technology authority requirements, further validation is required to determine whether data from countries other than China and the UK can be extrapolated to larger populations.

In conclusion, the present analysis indicates that the China Diabetes DSP, with appropriate weighting applied, is an epidemiologically valuable source of information on patients with T2DM that is representative of the wider diabetes population in China, as indicated by comparability of data collected in the DSP and a reference population-based cross-sectional survey. Comparison of the UK Diabetes DSP and a reference UK diabetes population derived from the HSFE provides further support for this approach in the diabetes setting. Together, these findings highlight the need for goodquality data collected using standardised collection methodologies and suggest that data generated using the DSP methodology may complement other data sources of information on patients with T2DM by filling a need for up-to-date patient treatment outcome data, which may ultimately inform public health decisionmaking in China.

Acknowledgements The authors thank Deirdre Carman for providing writing assistance and editorial support and Victoria Higgins and Andrea Leith of Adelphi Real World for reviewing the manuscript.

Collaborators Victoria Higgins and Andrea Leith.

Contributors SMB, BC, TH, GM and JP jointly designed the study and GM performed the statistical analyses. All authors participated in drafting and revising the manuscript. All authors read and approved the final manuscript.

Funding This work was supported by Eli Lilly \& Co. Adelphi Real World conducted these analyses with funding from Eli Lilly \& Co. Financial support for writing assistance and editorial support was supplied by Eli Lilly \& Co.

Competing interests SMB and BC are employed by and shareholders in Eli Lilly \& Co. TH, GM and JP are employed by Adelphi Real World.

Provenance and peer review Not commissioned; externally peer reviewed.

Open Access This is an Open Access article distributed in accordance with the Creative Commons Attribution Non Commercial (CC BY-NC 4.0) license, which permits others to distribute, remix, adapt, build upon this work noncommercially, and license their derivative works on different terms, provided the original work is properly cited and the use is non-commercial. See: http:// creativecommons.org/licenses/by-nc/4.0/

\section{REFERENCES}

1. IDF Diabetes Atlas Sixth Edition, update 2014. http://www.idf.org/ atlasmap/atlasmap?indicator $=i 1 \&$ date $=2014$ (accessed 28 May 2015).

2. National Diabetes Research Group. A mass survey of diabetes mellitus in a population of 300,000 in 14 provinces and municipalities in China (author's transl). Zhonghua Nei Ke Za Zhi 1981;20:678-83.

3. Diabetes in China: mapping the road ahead. Lancet Diabetes Endocrinol 2014;2:923.

4. Saunders C, Byrne CD, Guthrie B, et al. Scottish Diabetes Research Network Epidemiology Group. External validity of randomized controlled trials of glycaemic control and vascular disease: how representative are participants? Diabet Med 2013;30:300-8.

5. Ruokoniemi $P$, Sund R, Arffman M, et al. Are statin trials in diabetes representative of real-world diabetes care: a population-based study on statin initiators in Finland. BMJ Open 2014:4:e005402

6. Anderson $\mathrm{P}$, Benford $\mathrm{M}$, Harris $\mathrm{N}$, et al. Real-world physician and patient behaviour across countries: Disease-Specific Programmesa means to understand. Curr Med Res Opin 2008;24:3063-72.

7. EphMRA Code of Conduct. Updated January 2016. http://www. ephmra.org/code-of-conduct/2/A-Purpose-Scope-and-Sources (accessed 21 Apr 2016).

8. US Department of Health and Human Services. Summary of the HIPAA Privacy Rule May 2003. http://www.hhs.gov/sites/default/files/ privacysummary.pdf (accessed 21 Apr 2016).

9. Ji LN, Lu JM, Guo XH, et al. Glycemic control among patients in China with type 2 diabetes mellitus receiving oral drugs or injectables. BMC Public Health 2013;13:602.

10. Health and Social Care Information Centre. Health Survey for England-2011, health, social care and lifestyles. 20 Dec 2012. http://www.hscic.gov.uk/catalogue/PUB09300 (accessed 28 May 2015).

11. Health and Social Care Information Centre. Health Survey for England-2011, health, social care and lifestyles. List of variables. Version 1. http://doc.ukdataservice.ac.uk/doc/7260/mrdoc/pdf/ 7260datadocs.pdf (accessed 28 May 2015). 
12. Health and Social Care Information Centre. Health Survey for England-2011. Volume 2 Methods and documentation. 2012. http:// www.hscic.gov.uk/catalogue/PUB09300/

HSE2011-Methods-and-docs.pdf (accessed 28 May 2015).

13. Schuirmann DA. A comparison of the two one-sided tests procedure and the power approach for assessing the equivalence of average bioavailability. Pharmacometrics 1987;15:657-80.

14. Chow S-C, Shaeo J, Wang H, eds. Sample size calculations in clinical research. ISBN 0-8247-0970-5. New York (NY): Marcel Dekker, 2003:13.

15. Bi Y, Zhu D, Cheng J, et al. The status of glycemic control: a cross-sectional study of outpatients with type 2 diabetes mellitus across primary, secondary, and tertiary hospitals in the Jiangsu province of China. Clin Ther 2010;32:973-83.
16. Ji L, Li H, Guo X, et al. Impact of baseline BMI on glycemic control and weight change with metformin monotherapy in Chinese type 2 diabetes patients: phase IV open-label trial. PLoS One 2013;8:e57222.

17. Guo XH, Yuan L, Lou QQ, et al. Chinese Diabetes Education Status Survey Study Group. A nationwide survey of diabetes education, self-management and glycemic control in patients with type 2 diabetes in China. Chin Med J (Engl) 2012;125:4175-80.

18. NHS Diabetes Working Group. Self-monitoring of blood glucose in non-insulin-treated type 2 diabetes. 2010. http://webarchive. nationalarchives.gov.uk/20130513172055/http:/www.diabetes.nhs. uk/document.php?o=238 (accessed 28 May 2015).

19. Yasky J, Verho M, Erasmus TP, et al. Efficacy of ramipril versus enalapril in patients with mild to moderate essential hypertension. Br J Clin Pract 1996;50:302-10. 